\title{
Observer Based Temperature Control for Reduced Thermal Cycling in Power Electronic Cooling
}

\author{
Xiang Wang, Alberto Castellazzi, Pericle Zanchetta \\ The Power Electronics, Machines and Control (PEMC) Research Group, Electrical \\ Systems and Optics Research Division, Department of Electrical and Electronic \\ Engineering, University of Nottingham, Nottingham, NG7 2RD, United Kingdom (UK).
}

\begin{abstract}
This paper presents an advanced dynamic cooling strategy for multi-layer structured power electronic modules. A observer based feedback controller is proposed to reduce a power device or module's thermal cycle amplitude during operation, with the aim of improving reliability and lifetime. The full-state observer design is based on a developed Cauer type thermal model. The observer enables estimation and control of the temperature at reliability critical locations only measuring one accessible location. This makes the method particularly powerful and suitable for application in power systems. The designed strategy is confirmed experimentally. Although the experiment is developed for a specific application scenario, the proposed strategy is of general validity.
\end{abstract}

Keywords: Cooling, Reliability, Temperature Control, Thermal Stress, Thermal Cycle

\section{Introduction}

A typical packaged semiconductor power switch or module has multilayer structure, and the schematic is shown in Fig. 1. The different materials used in this multilayer structure provide different thermal expansion coefficients for each layer [1]. During operation, temperature cycles lead to different

Email addresses: eexxw13@nottingham.ac.uk (Xiang Wang), alberto.castellazzi@nottingham.ac.uk (Alberto Castellazzi), pericle.zanchetta@nottingham.ac.uk (Pericle Zanchetta) 
thermo-mechanical stresses between adjacent layers, which leads to degradation or even failure of the module.

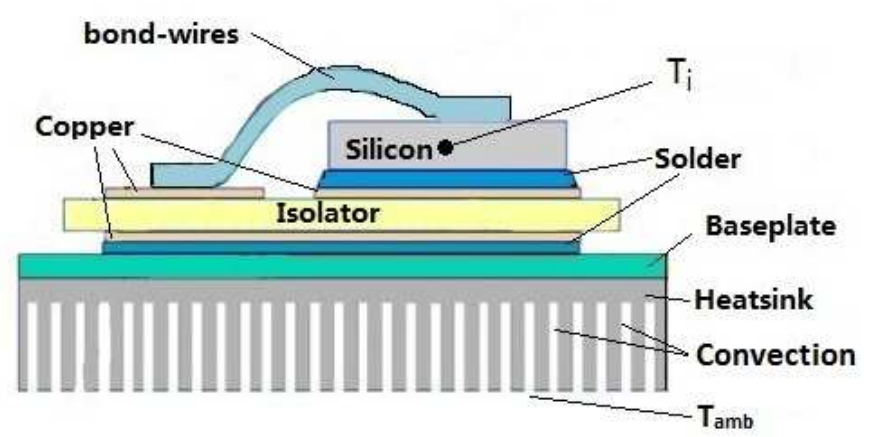

Figure 1: Semiconductor power module structure

The failure mechanisms generally can be grouped by random and wearout failures $[2,3,4]$. Wear-out mechanism failures make up the majority of failures in power electronic modules [5]. In wear-out mechanism, thermomechanical stress plays a very important role in affecting power electronic devices/modules reliability, such as the fractures propagation and degradations in solder layers, wire-bond lift-off and emitter metallization $[6,2,7,8,9]$. Fig. 2 (a) shows an acoustic microscope image of the baseplate of a power module, revealing solder layer delamination below the substrates; Fig. 2 (b) shows an example of wire-bond lift-off after power cycling of a module.

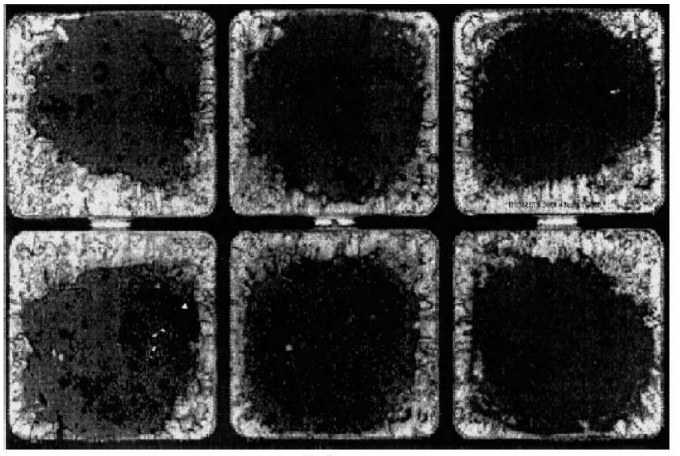

(a)

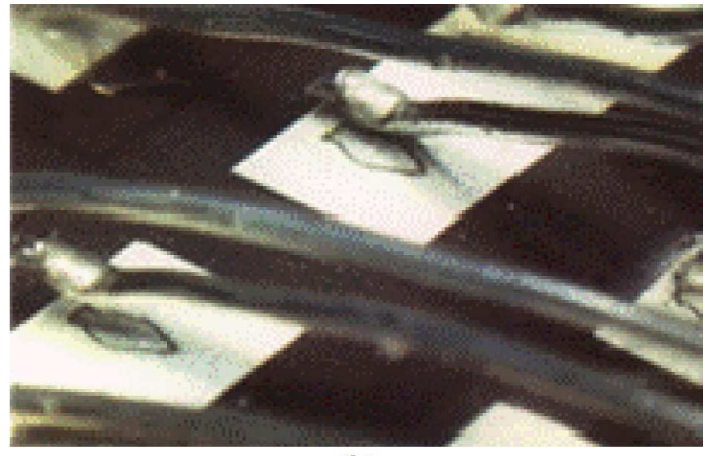

(b)

Figure 2: Typical failure mechanisms of semiconductor power electronic modules: (a) solder layer delamination; b)bond-wire lift-off

The failure mechanisms are influenced by both environmental and load conditions $[10,11]$. To address this issue, research has addressed different 
aspects, for example, new semiconductor and materials technologies [12, 13], package architecture [14], interconnection [15], control of power electronic modules [16] and advanced cooling technologies [6, 17]. The motivation for this work is based on a more detailed analysis of Fig. 3 showing a summary of the results of extensive reliability tests on IGBT power modules [18]. These results clearly indicated that a power module's operational lifetime depends mainly on the average operational temperature, Tm, and the amplitude of the thermal cycles, $\Delta \mathrm{T}$, that the module experiences $[2,18]$. From Fig. 3 one can see that if $\Delta \mathrm{T}$ is reduced by even the same amount that $\mathrm{Tm}$ is increased, a much higher number of cycles to failure can be achieved. For instance, moving from point 1 to point 2 , As $\Delta \mathrm{T}$ is fixed at $50 \mathrm{~K}$, increasing $\mathrm{Tm}$ by $20 \mathrm{~K}$ from $80^{\circ} \mathrm{C}$ to $100^{\circ} \mathrm{C}$, the cycles to failure will be reduced $3 \times 10^{5}$ cycles from point $1\left(2 \times 10^{5}\right.$ cycles $)$ to point $2\left(5 \times 10^{5}\right.$ cycles $)$. However, moving from point 2 to point 3 , keeping the same Tm, a reduction of $20 \mathrm{~K}$ in $\Delta \mathrm{T}$ increases the number of cycles to failure back to $2 \times 10^{6}$, that is, even better than the starting point 1.

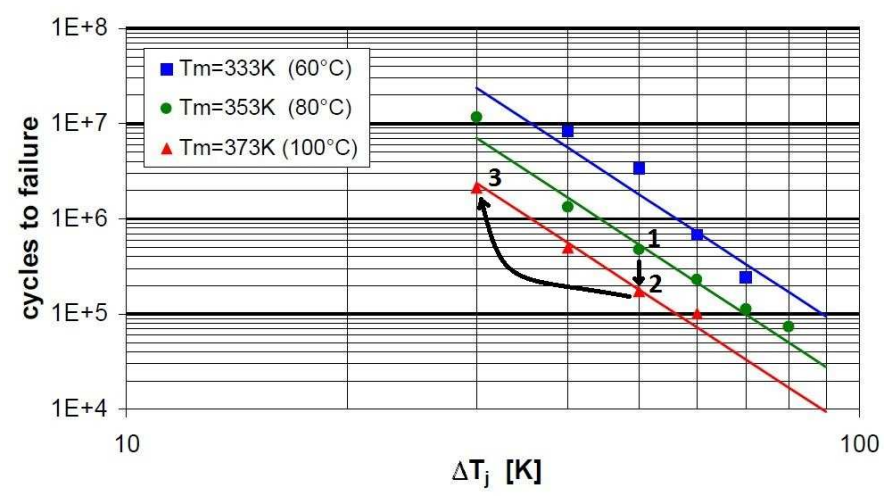

Figure 3: Reliability of power modules as a function of thermal cycle amplitude for different values of the average temperature [18]

However, typical power device thermal management design only aims to ensure that the maximum operating temperature is kept below a safety critical value at full-load or worst-case conditions and the cooling device is based on fixed designed parameters. This is not optimum for thermal stresses consideration nor energy saving. Therefore, considering lifetime and energy efficiency, the adaptive cooling strategy is proposed here. As shown in Fig. 4, the temperature with constant cooling power will vary as load changes. In order to decrease $\Delta \mathrm{T}$, the cooling power can be adjusted according to the load 
variations and this can be achieved simply by reducing the cooling power.

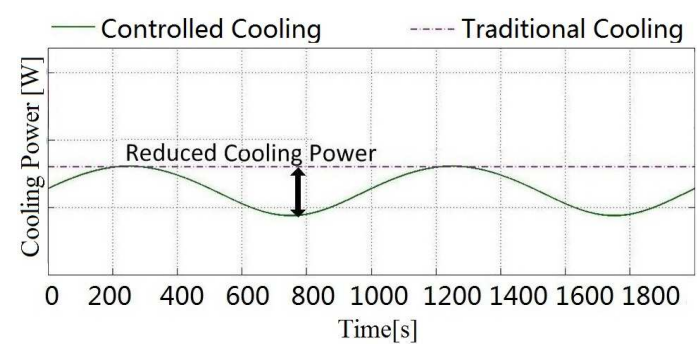

(a)

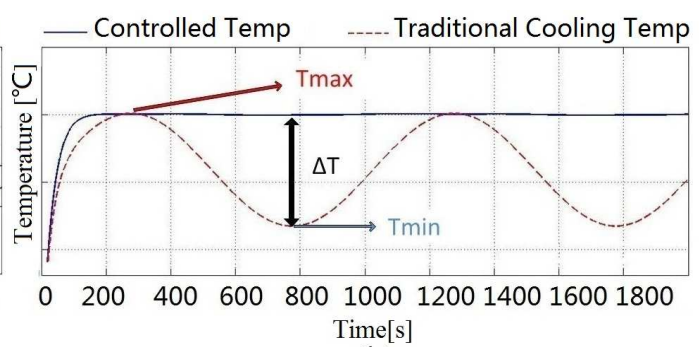

(b)

Figure 4: Change in thermal cycling with traditional cooling approach and with proposed cooling strategy: (a) Cooling power (b) Temperature

In power electronics, thermal stresses are mainly lead by active and passive thermal cycling. Active thermal cycling is a result of power dissipation in the semiconductor devices. Therefore it is characterised by relatively high frequency components (typically a superposition of PWM (Pulse-width modulation) and load characteristic frequencies) and affects wire-bond lifetime. Passive thermal cycling is due to variations in ambient temperature and cooling conditions, and it usually takes place on large time-scales. Passive cycling mainly affects solder layers and baseplate reliability. However, active cycling has never been detected without passive cycling and passive cycling is also quite critical in many applications (e.g., large power systems). In reality, power devices, like converters used in traction or wind turbines, usually suffer both cycling modes and this results in different levels of temperature swings, as well as frequency [9].

In this work, the proposed dynamic active cooling controller is proposed, which is shown as in Fig. 5, where the temperature at the reliability critical location of the power assembly is controlled according to the actual load $P_{\text {diss }}$ (i.e., power dissipation) and boundary condition $T_{a m b}$ (i.e., ambient temperature). The feedback control loop monitors the temperature of the desired location $T_{\text {out }}$ and intervenes on the cooling parameter $V_{\text {cooling }}$ to eliminate temperature errors $T_{\text {err }}$ to control the temperature output and decrease temperature variations. The control parameter $V_{\text {cooling }}$ is the controller output signal used to control the cooling devices. It can be the bias voltage applied on the fan for a forced air convection cooling, or the voltage on the pump in a liquid cooling system. By controlling the cooling device, the thermal 
impedance of the system can be adjusted to meet the temperature regulation. In particular, in view of typical practical difficulties in introducing temperature sensors in the assembly of a packaged power device or module, the design has been formalized in the framework of a full-order observer state-space thermal model, so as to enable estimation and control of the temperature at reliability critical locations (e.g., substrate solder layer in a power module) based only on the actual temperature measurement at a different, more accessible location in the assembly (e.g.,the top of the heat-sink).

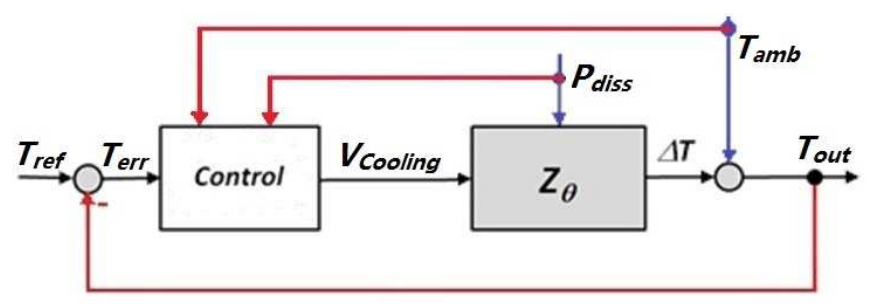

Figure 5: Block diagram for control design

Considering that both the control signal, $V_{\text {cooling }}$, and the disturbances, $P_{\text {diss }}$ and $T_{a m b}$, have impacts on the system, the State Feedback Control method is applied in a MIMO (Multiple Input Multiple Output) system. The developed design was tested in simulation and implemented experimentally in an DSP (Digital Signal Processor) platform. The results clearly show that temperature control can greatly reduce the amplitude of temperature variations resulting from variations in the power dissipation and ambient temperature profiles.

\section{Theory and Design}

\subsection{State-Space Cooler Control System Design}

A common modelling technique for power semiconductor thermal performance consists in the use of an equivalent essentially 1-D linear thermal model. For most cooling conditions and assembly structures, heat conduction can be assumed to take place essentially in the vertical direction, from the chips towards the heat-sink, enabling for an essentially 1-D treatment of the problem. This allows us to derive the associated thermal resistance, $R_{t h_{j}}$, and thermal capacitance, $C_{t h_{j}}$, of each layer, as schematically indicated in Fig. 6 (a) and Equation $(1,2)$. The thickness, $d_{j}$, of each layer is chosen 


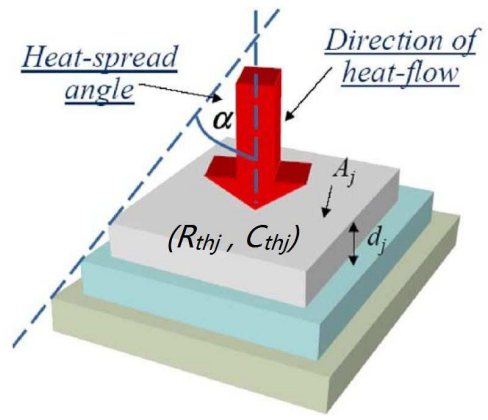

(a)

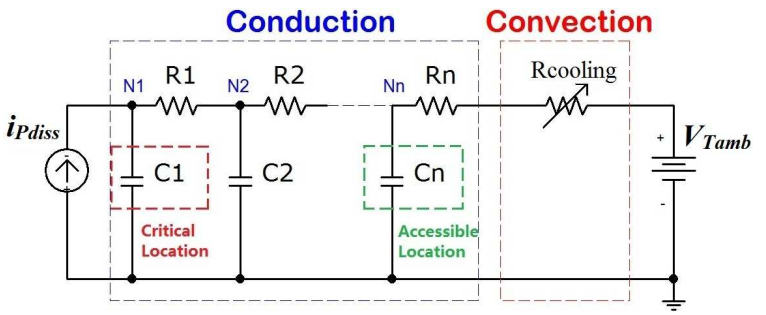

(b)

Figure 6: (a)Physical approach used for extracting the thermal impedance [19]; (b)Electrical equivalent circuit of the assembly

to correspond to the various physical regions of interest that can be identified inside the devices. As it is usual in modelling thermal effects with an approximated quasi-1-D heat-flow description, the lateral dimensions of each layer $A_{j}$ are derived starting from a given surface-active area and assuming an angle of $45^{\circ}$ for the spreading of the heat wave [19].

$$
\begin{gathered}
R_{t h_{j}}=\frac{d_{j}}{\lambda \cdot A_{j}} \\
C_{t h_{j}}=c \cdot \rho \cdot d_{j} \cdot A_{j}
\end{gathered}
$$

where:

$$
\begin{gathered}
\lambda=\text { thermal conductivity; } \\
A_{j}=\text { surface area; } \\
\rho=\text { density; } \\
c=\text { specific heat capacity; } \\
d_{j}=\text { thickenss of step length }
\end{gathered}
$$

Considering that geometrical structure based models can ensure a high precision and validity over a wide range of device operation conditions [20], we created, as shown in Fig. 6 (b), an Cauer-type electrical equivalent circuit for the assembly including cooling device. In the circuit structure shown in Fig. 6 (b), each node of the network corresponds to an actual physical location of the assembly: voltage corresponds to temperature and current to heatflow. Here, the convective boundary conditions are emulated by means of a 
variable thermal resistance, $R_{\text {cooling }}$, which corresponds to the heat-sink and convection part shown in Fig. 1. The thermal resistances and capacitances values in Fig. 6 (b) can be calculated by applying Equation $(1,2)$. The action of the convection cooling is modelled by Equation (3), where the cooler's thermal resistance, $R_{\text {cooling }}$, is a function of control parameters (e.g., the bias voltage of a fan or that of a liquid cooler's pump). In our design, $R_{\text {cooling }}$ becomes the control variable and it will be adjusted during the operation.

$$
\Phi=\frac{T_{n}-T_{a m b}}{R_{n}+R_{\text {cooling }}}
$$

The derived thermal model can be formalised in the framework of statespace description: Equation (4)

$$
\begin{aligned}
& \dot{\bar{x}}=A \cdot \bar{x}+B \cdot \bar{u} \\
& \bar{y}=C \cdot \bar{x}+D \cdot \bar{u}
\end{aligned}
$$

Here the system states are the temperature values at each nodes,

$$
\bar{x}=\left[\begin{array}{lllll}
V_{C_{1}} & V_{C_{2}} & \cdots & V_{C_{n-1}} & V_{C_{n}}
\end{array}\right]^{T}
$$

and the system inputs shown in Equation (6) are: the control signal $V_{\text {cooling }}$, the disturbance power dissipation $P_{\text {diss }}$ and the boundary condition ambient temperature $T_{a m b}\left(i_{P_{\text {diss }}}\right.$ and $V_{T_{a m b}}$ will be replaced with $P_{\text {diss }}$ and $T_{a m b}$ for expression clarity).

$$
\bar{u}=\left[P_{\text {diss }} V_{\text {cooling }} T_{\text {amb }}\right]^{T}
$$

The system output temperature are chosen as the node temperatures:

$$
\bar{y}=\bar{x}
$$

Based on the derived equivalent thermal model in Fig. 6 (b), the differential description can be derived according to Kirchhoff's Current Law as the 
following:

$$
\begin{gathered}
i_{P_{\text {diss }}}=i_{R_{1}}+i_{C_{1}}=\frac{V_{C_{1}}-V_{C_{2}}}{R_{1}}+C_{1} \cdot \dot{V}_{C_{1}} \\
i_{R_{1}}=i_{R_{2}}+i_{C_{2}}=\frac{V_{C_{2}}-V_{C_{3}}}{R_{2}}+C_{2} \cdot \dot{V}_{C_{2}} \\
\vdots \\
i_{R_{n-2}}=i_{R_{n-1}}+i_{C_{n-1}}=\frac{V_{C_{n-1}}-V_{C_{n}}}{R_{n-1}}+C_{n-1} \cdot \dot{V}_{C_{n-1}} \\
i_{R_{n-1}}=i_{R_{n}}+i_{C_{n}}=\frac{V_{C_{n}}-V_{T_{\text {amb }}}}{R_{n}+R_{\text {cooling }}}+C_{n} \cdot \dot{V}_{C_{n}}
\end{gathered}
$$

Equation (8) can be re-arranged as shown in Equation (9)

$$
\begin{aligned}
\dot{V}_{C_{1}}= & V_{C_{1}} \cdot \frac{-1}{C_{1} \cdot R_{1}}+V_{C_{2}} \cdot \frac{1}{C_{1} \cdot R_{1}}+i_{P_{\text {diss }}} \cdot \frac{1}{C_{1}} \\
\dot{V}_{C_{2}}= & V_{C_{1}} \cdot \frac{1}{C_{2} \cdot R_{1}}-V_{C_{2}} \cdot\left(\frac{1}{C_{2} \cdot R_{1}}+\frac{1}{C_{2} \cdot R_{2}}\right)+V_{C_{3}} \cdot \frac{1}{C_{2} \cdot R_{2}} \\
\vdots= & \vdots \\
\dot{V}_{C_{n-1}}= & V_{C_{n-2}} \cdot \frac{1}{C_{n-1} \cdot R_{n-2}}-V_{C_{n-1}} \cdot\left(\frac{1}{C_{n-1} \cdot R_{n-2}}+\frac{1}{C_{n-1} \cdot R_{n-1}}\right) \\
& +V_{C_{n}} \cdot \frac{1}{C_{n-1} \cdot R_{n-1}} \\
\dot{V}_{C_{n}}= & V_{C_{n-1}} \cdot \frac{1}{C_{n} \cdot R_{n-1}}-V_{C_{n}} \cdot\left(\frac{1}{C_{n} \cdot R_{n-1}}+\frac{1}{\left(C_{n} \cdot R_{n}\right)+C_{n} \cdot R_{\text {cooling }}}\right) \\
& +V_{T_{a m b}} \cdot \frac{1}{\left(C_{n} \cdot R_{n}\right)+C_{n} \cdot R_{\text {cooling }}}
\end{aligned}
$$

then the system description in Equation (9) can be matched to Equation (4), and the model matrices described as in Equation (10,11,12,13.

$A=$

$$
\left(\begin{array}{ccccccc}
-\frac{1}{C_{1} \cdot R_{1}} & \frac{1}{C_{1} \cdot R_{1}} & 0 & \ldots & 0 & 0 & 0 \\
\frac{1}{C_{2} \cdot R 1} & -\frac{1}{C_{2} \cdot R_{1}}-\frac{1}{C_{2} \cdot R_{2}} & \frac{1}{C_{2} \cdot R 2} & \ldots & 0 & 0 & 0 \\
0 & 0 & 0 & \cdots & \frac{1}{C_{n-1} \cdot R n-2} & -\frac{1}{C_{n-1} \cdot R_{n-2}}-\frac{1}{C_{n-1} \cdot R_{n-1}} & \frac{1}{C_{n-1} \cdot R n-1} \\
0 & 0 & 0 & \ldots & 0 & \frac{1}{C_{n} \cdot R_{n-1}} & -\frac{1}{C_{n} \cdot R_{n-1}}-\frac{1}{d e n}
\end{array}\right)
$$




$$
B=\left(\begin{array}{ccc}
\frac{1}{C_{1}} & 0 & 0 \\
0 & 0 & 0 \\
\vdots & \vdots & \vdots \\
0 & \frac{\left(T_{n} \cdot C n-T_{a m b} \cdot C_{n}\right) \cdot n u m}{d e n^{2}} & \frac{1}{d e n}
\end{array}\right)
$$

where $n u m=\frac{d R_{\text {cooling }}}{d V_{\text {cooling }}}, \quad$ den $=C_{n} \cdot R_{n}+C_{n} \cdot R_{\text {cooling }}$,

$$
C=\boldsymbol{I}
$$

where $\boldsymbol{I}$ is the unity matrix,

$$
D=\boldsymbol{O}
$$

where $\boldsymbol{O}$ is the zero matrix.

In the state-space system, among the inputs, only $V_{\text {cooling }}$ is the controllable signal, and both $P_{\text {diss }}$ and $T_{a m b}$ are decided by the loading and the external environment so that they can be treated as system disturbances. To control the output temperature reaching the desired temperature value accurately, the system output temperature should has zero steady-state error to a step command. However, using the multivariable state feedback control on its own, any change in the model parameters will cause the error to be non-zero. Therefore, integral control is introduced by adding the integral of the system error to Equation (4). For the system described by Equation (4), one can feed back the integral of the error, $T_{\text {err }}=T_{\text {out }}-T_{\text {ref }}$, as well as the state of the plant, $\bar{x}$, by augmenting the plant state with the extra integral state $x_{I}$ as shown in Fig. 7 .

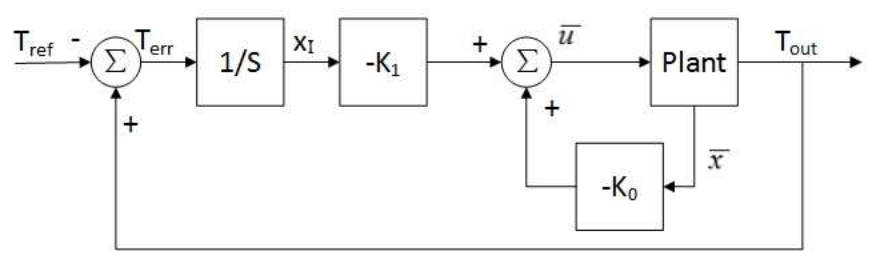

Figure 7: Augmented system

The open-loop system eigenvalues (poles) of the augmented system can be calculated and then the closed-loop poles can be placed according to the desired dynamic response performance by the feedback law 


$$
\bar{u}=-\left[\begin{array}{ll}
K_{1} & K_{0}
\end{array}\right]\left[\begin{array}{c}
x_{I} \\
\bar{x}
\end{array}\right]
$$

or simply

$$
\bar{u}=-K\left[\begin{array}{c}
x_{I} \\
\bar{x}
\end{array}\right] .
$$

In semiconductor power devices, it is difficult to measure and control temperature at a location inside the packaged power modules. One can measure the temperature at the accessible interface between baseplate and heat-sink and control the temperature at the desired critical location by the help of a system observer. Since the system states are observable, the fullorder observer can be built in the form of Equation. (14).

$$
\begin{aligned}
& \dot{\overline{\hat{x}}}=A \cdot \hat{\bar{x}}+B \cdot \bar{u}+L \cdot(\bar{y}-\hat{\bar{y}}) \\
& \hat{\bar{y}}=C \cdot \hat{\bar{x}}+D \cdot \bar{u}
\end{aligned}
$$

where $\mathrm{L}$ is the observer feedback matrix.

The observer error will decay to zero according to the eigenvalues of $(A-L C)$. Since the aim is to let the estimated state $\hat{x}$ follow the real state $x$ quickly, any error between them must decay faster than the closed loop transient. Therefore, the slowest eigenvalue of $(A-L C)$ is chosen to be an order of magnitude larger than the dominant closed-loop poles. The combined observer based feedback controller structure is shown in Fig. 8.

\subsection{Case Study}

For illustration, in this paper we refer specifically to the case of an aircooled forced-convection heat-sink, where the heat-transfer coefficient is determined by the applied bias voltage (i.e., the rotation speed) of the cooling fan. In this study we will use a simplified power assembly consisting of power resistors mounted on a fan-cooled heat sink instead of a real power module for ease of thermal modelling. The thermal power resistors will generate heat when dissipating power in the assembly therefore raising its temperature. The assembly is shown in Fig. 12. Through Equation $(1,2)$ the thermal resistance and thermal capacitance can be calculated, where the step length, $d_{j}$, along the heat sink is chosen following that the subsequent layer step length is double the previous one. 


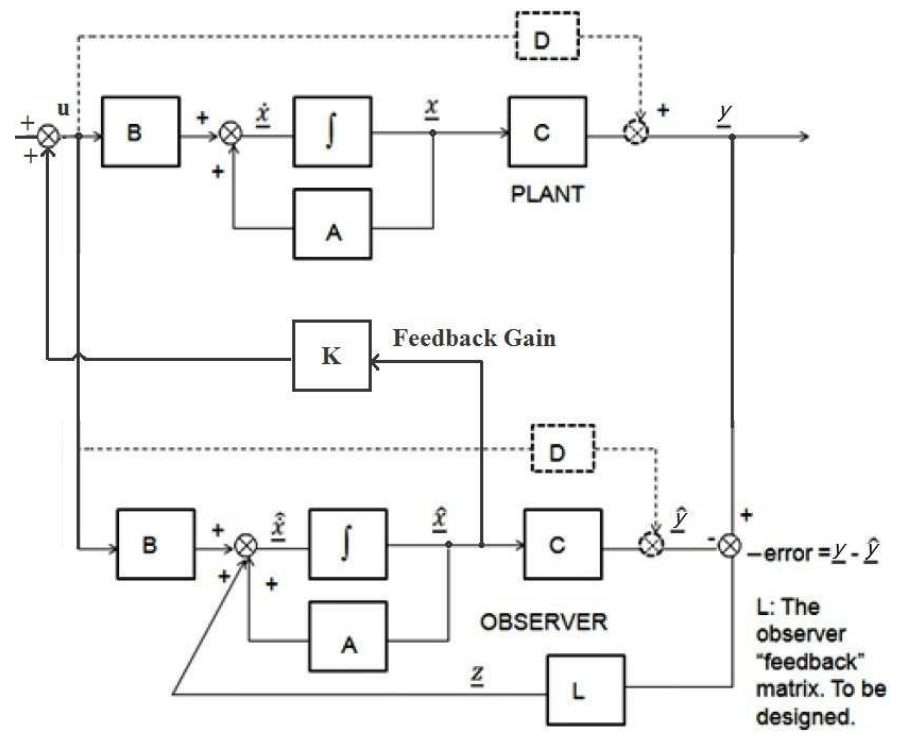

Figure 8: Observer based feedback control structure

In this specific air-cooled forced-convection prototype case, the controllable thermal resistance, $R_{\text {cooling }}$, and the bias voltage applied on the cooling fan, $V_{\text {cooling }}$, have the relation shown in Equation (15), which is derived from the information and data-sheet provided by the manufacturer.

$$
\begin{aligned}
R_{\text {cooling }}= & 0.00114846 \cdot\left(0.277170 \cdot V_{\text {cooling }}+0.081015\right)^{4} \\
& -0.01837536 \cdot\left(0.277170 \cdot V_{\text {cooling }}+0.081015\right)^{3} \\
& +0.12001407 \cdot\left(0.277170 \cdot V_{\text {cooling }}+0.081015\right)^{2} \\
& -0.1205101387 \cdot V_{\text {cooling }}+0.8986650552
\end{aligned}
$$

Because of the non-linear characteristics in Equation (15), the model needs to be linearised so that classic modern control methods can be applied to achieve the expected control performances. We can plot Equation (15), see Fig. 9, and we can see that this curve is meaningful only when $V_{\text {cooling }}$ ranges from $0 \mathrm{~V}$ to $20 \mathrm{~V}$ because the higher bias voltage means higher cooling power which should result in lower thermal resistance instead of the increasing thermal resistance. Considering that the curve shown in Fig. 9 is smooth and has a continuous derivative, the Small-Signal Linearisation method [21] can be used here and the equilibrium point in terms of $V_{\text {cooling }}$ for linearisation should be chosen within the operational range ( here is $0 \mathrm{~V}$ to $20 \mathrm{~V}$ ). 


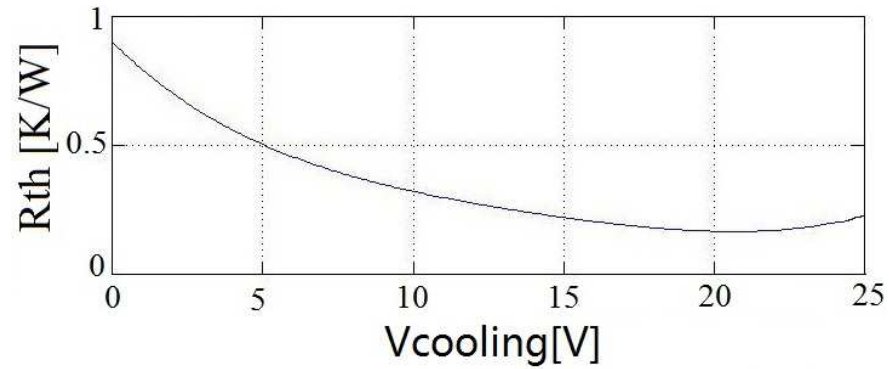

Figure 9: The characteristics of thermal resistance to bias voltage of the fan

The working conditions in this paper are: $P_{\text {diss }}=100-200 \mathrm{~W} ; V_{\text {cooling }}=$ $2-20 \mathrm{~V} ; T_{a m b}=20^{\circ} \mathrm{C}$, for which experimental results are available. Because the definition of the equilibrium point is a point $\mathrm{O}$ which satisfies $\dot{x}_{o}=0=$ $f\left(x_{o}, u_{o}\right)$, any stable operating points could be chosen as this equilibrium point. However, considering that the system load (power dissipation and ambient temperature) normally won't be fixed at a certain point during the operation together with the presence of unavoidable linearisation errors, we should choose an operating point producing the lowest effective error. The linearisation is only in terms of $V_{\text {cooling }}$ and therefore the operating point of linearisation mainly depends on $V_{\text {cooling }}$, which will range within $20 \mathrm{~V}$ for different load conditions. Simulations of different output temperature values at various cooling voltage levels have been done at different operating points. The errors between a linearised state-space system at certain operating point and the original system are then compared and calculated with Equation (16) as shown in Fig. 10.

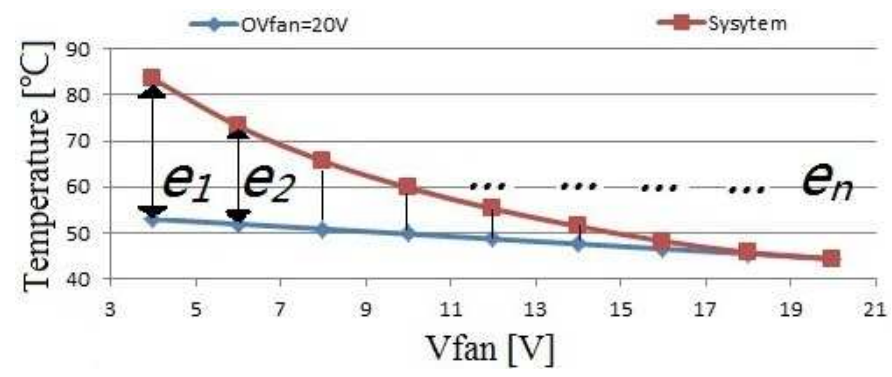

Figure 10: Error calculation 


$$
\begin{aligned}
E & =\sqrt{\frac{e_{1}^{2}+e_{2}^{2}+e_{3}^{2}+\ldots+e_{n}^{2}}{n}} \\
e_{n} & =T_{n}-T_{\text {system }}
\end{aligned}
$$

Following calculations $V_{\text {cooling }}=16 \mathrm{~V}$ has been chosen as the best operating point for its smallest effective error compared to other operating points. Next, the state-space model is linearised around the system operational point, $P_{\text {diss }}=150 \mathrm{~W}, V_{\text {cooling }}=16 \mathrm{~V}$ and $T_{a m b}=20^{\circ} \mathrm{C}$.

Then, by applying the derived system parameters and operating point into the state-space model described in Equation (4), the augmented system open-loop eigenvalues (poles) can be calculated and the results can be seen in Table. 1.

Table 1: System Eigenvalues and Feedback Gains

\begin{tabular}{cccc}
\hline \hline System states & Open-loop & Closed-loop & Feedback Gains \\
\hline 7 & 0 & $-0.063+0.03 \mathrm{i}$ & -0.7596 \\
6 & -5978.30 & -5978.30 & -47.9764 \\
5 & -1141.51 & -1141.51 & 166.4610 \\
4 & -254.22 & -254.22 & -129.5699 \\
3 & -59.43 & -59.43 & 29.3406 \\
2 & -11.43 & -11.43 & 91.2576 \\
1 & -0.038 & $-0.063-0.03 \mathrm{i}$ & -136.6341 \\
\hline
\end{tabular}

Since in the augmented system we can identify two dominant poles in open-loop, while other poles are far away from them, it is sufficient to place only these two poles and change them in the closed loop configuration while leaving all other unchanged.

By choosing the system damping ratio equal to 0.9 (that produced by the two dominant poles) to avoid overshoot. The settling time is chosen equal to 70 seconds according to the system time constant. The two dominant closed loop poles have been selected. A pole-placement philosophy that aims to fix only the undesirable aspects of the open-loop response and avoids either large increases in bandwidth or efforts to move poles that are near zeros will typically allow smaller gains, and thus smaller control actuators, than a philosophy that arbitrarily picks all the poles without regard to the original 
open-loop pole and zero locations [21]. Therefore, the non-dominant poles remain in their original position. The corresponding feedback gain values were extracted automatically in Matlab with the dedicated command for closedloop pole placement. The specific closed-loop poles and the corresponding feedback gains are also shown in Table.1.

The Selected Observer Eigenvalues are shown in Table.2 and the observer gain shown in Equation (17).

Table 2: Observer Gains

\begin{tabular}{ccc}
\hline \hline System states & System Eigenvalues & Observer Eigenvalues \\
\hline 6 & -5978.30 & -900 \\
5 & -1141.51 & -800 \\
4 & -254.22 & -700 \\
3 & -59.43 & -600 \\
2 & -11.43 & -500 \\
1 & -0.038 & -400 \\
\hline
\end{tabular}

$$
L=\left(\begin{array}{cccccc}
-3345.24 & 3745.24 & 0 & 0 & 0 & 0 \\
1852.96 & -2289.27 & 936.31 & 0 & 0 & 0 \\
0 & 458.46 & -92.53 & 234.077 & 0 & 0 \\
0 & 0 & 112.31 & 529.17 & 58.52 & 0 \\
0 & 0 & 0 & 27.01 & 758.36 & 14.63 \\
0 & 0 & 0 & 0 & 5.34 & 894.61
\end{array}\right)
$$

By only measuring the temperature at the typical accessible location, with the help of full-order observer, the controller can still control the temperature $T_{\text {out }}$ at the critical location and track the reference temperature precisely.

\section{Experiment and Hardware Development}

Considering the task of real-time signal processing and further controlalgorithm implementation, DSP microprocessor was used here. DSP has been applied in various areas such as communications, controls and image processing, because the have become increasingly cost-effective [22]. Realtime processing requires the processing must keep pace with some external 
event which is usually the analog input. The sensed signals (Power dissipation $P_{\text {diss }}$, ambient temperature $T_{a m b}$ and output temperature $T_{\text {out }}$ ) are transformed into voltage signals, sent through an AD (analog to digital) converter to the FPGA and then stored in the memory. DSP processor reads the digital signals through the memory and sends the calculated data back to the FPGA. The DA (digital to analog) converter transforms the digital signal into analog mode and sends them to the drive circuit to drive the cooler. All of the regulation and control calculations are processed inside the programmed DSP chip. The FPGA system used in this research is also in charge of pulse generation, signal reading, data sampling, data conversion, and incorporation with external interface boards.

The heat source (power electronic modules)was placed on the top of the heat-sink, and the temperature sensors are located in the positions as shown in Fig. 11. The assembly will be placed inside a thermal chamber to provide various ambient temperature environment. Although we are only measuring the temperature at the typical accessible location, with the help of full-order observer, the controller can still control the temperature $T_{\text {out }}$ at the critical location to track the reference temperature precisely. In Fig. 11, the temperature at the accessible location $T_{\text {measure }}$ is sensed and sent to the observer-based controller to control the temperature $T_{\text {out }}$ at the critical location without direct measuring. The simple assembly of the whole system is shown in Fig. 12.

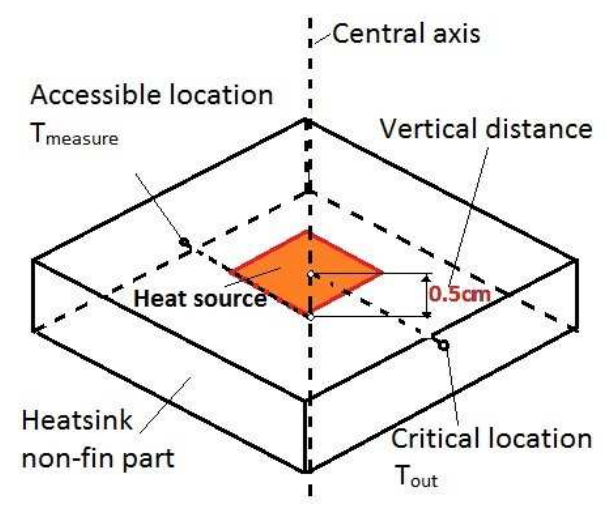

Figure 11: Sketch graph for temperature location

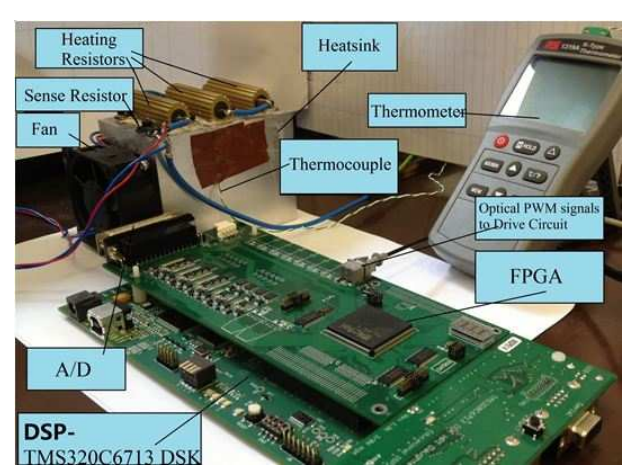

Figure 12: Overall hardware set-up for experimental test

In this research, both of the two temperature signals are sensed by K type 
thermocouples. Thermocouples are a good choice because they are small in size, have long-term stability and fast response.

To drive a fan there are different possible solutions, for example PWM methods or linear regulation. The current trend in electronic enclosure cooling is to regulate the cooling fan/blower speed according to the temperature inside the enclosure. PWM is the preferred method to regulate motor speed because no additional heat is generated, and it is energy efficient is higher when compared to linear regulating (voltage control) motor [6]. The linear relation between the equivalent output voltage and Duty-cycle ensures the designed controller can be applied in driving the fan with either PWM method or linear regulation [6]. The PWM duty cycle is defined as $T_{\text {on }} / T_{\text {off }}$ in one period and the range is $0-1$ and the PWM driving signal $V_{G S}$ used in our experiments is shown in Fig. 13. As seen from this picture, the fan is driven by turning on/off of the gate signals in PWM mode: on-state and off-state (15 Volts and 0 Volts). The cooling fan speed will be tuned via the adjustment of the duty cycle. Although the PWM frequency has no influence on tuning the fan speed, different frequencies will result in different power consumptions for the drive circuit. Therefore, a reasonable PWM frequency should be chosen to maintain good performance with minimum power consumption. Fig. 14 shows the experimental measured power consumption corresponding to different PWM switching frequencies.

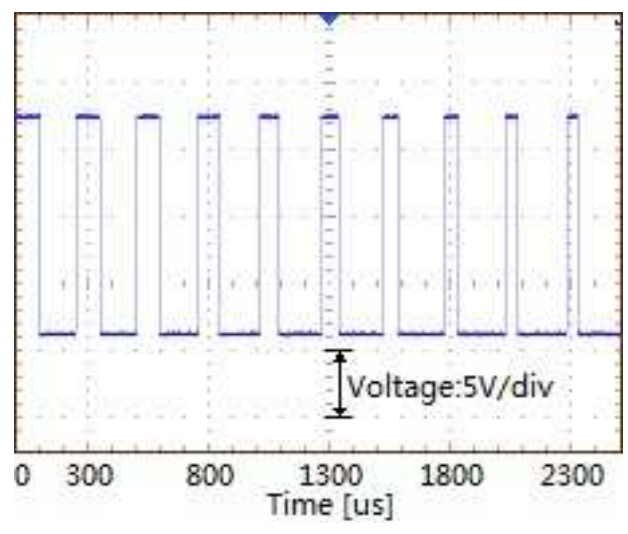

Figure 13: PWM driving signal $\left(V_{G S}\right)$

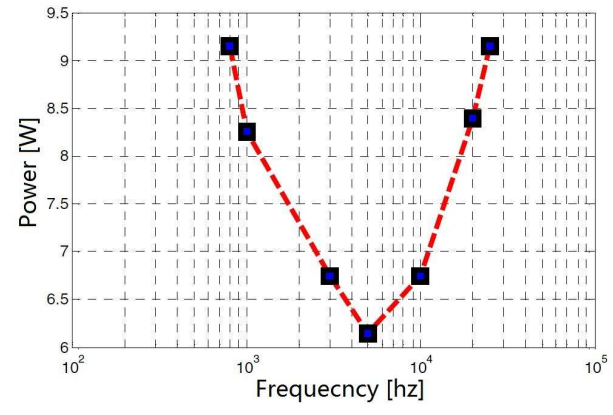

Figure 14: Power consumption for different PWM frequencies

From Fig. 14 we can see that when PWM switches at $5 \mathrm{kHz}$ the lowest power consumption of the drive circuit can be achieved. Therefore, $5 \mathrm{kHz}$ switching frequency was applied to minimize the switching power losses, with 
a consideration that frequency below $5 \mathrm{kHz}$ may also lead to relatively worse acoustic performance.

\section{Experimental Implementation and Results}

The designed cooler test under continuous various load conditions has been done experimentally. The test load condition (power dissipation and ambient temperature) profiles are shown in Fig. 15 (a). The corresponding temperature results are shown in Fig. 15 (b). Comparing to the cooling under fixed nominal cooling power, the designed feedback controller is extremely effective in reducing the amplitude of temperature cycles (e.g., $60^{\circ} \mathrm{C}$ in this test), and almost eliminates the variation for varying power dissipation and ambient temperature profiles. Therefore the contribution to extending operation lifetime can be made for multi-layer structured power devices and modules. Noting that Fig. 15 (a) only shows the power dissipation $(100 \mathrm{~W}$ to $200 \mathrm{~W})$ and ambient temperature $\left(-25^{\circ} \mathrm{C}\right.$ to $\left.+25^{\circ} \mathrm{C}\right)$ running at a frequency of $0.001 \mathrm{~Hz}$ and $0.0002 \mathrm{~Hz}$ respectively, but different frequency combinations have also been tested and receive very similar, good results. The results for different frequency loads are tested and shown in Fig. 16.

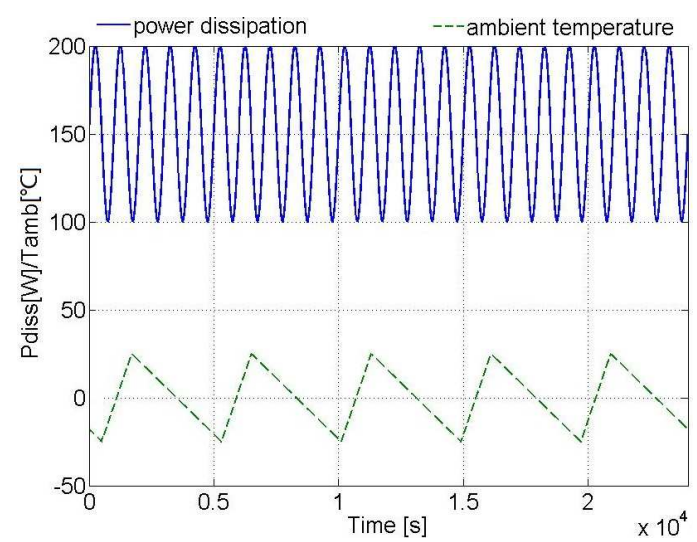

(a)

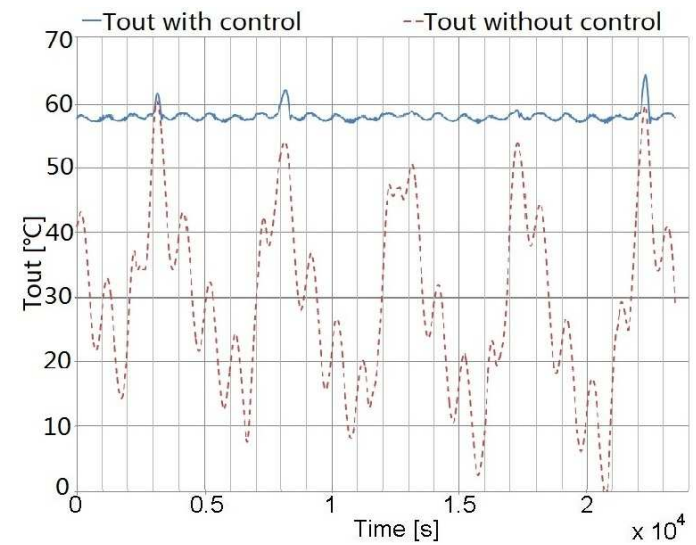

(b)

Figure 15: (a)Experimental test conditions; (b)Experimental results

In the frequency domain test, a sinusoidal power DC supply was used to generate power dissipation profiles ranging from $100 \mathrm{~W}$ to $200 \mathrm{~W}$ under different frequencies to heat the power resistor. Fig. 16 shows the observer based 
controller's performance in terms of reduction in $\delta T$ (temperature variation) and increase in $T_{m}$ (average temperature). The temperature under the designed controller was compared with the nominal fixed cooling power. As we discussed in Fig. 3, in this picture, as long as the curve of $\delta T$ is higher than the $T_{m}$ curve, i.e. in left side of the crossing point $P_{\text {crossing }}$, a meaningful improvement in reliability in terms of cycles to failure can be achieved. In this specific case study this occurs as long as the load profile varies within the frequency below the cross point $(0.004 \mathrm{~Hz})$ in Fig. 16.

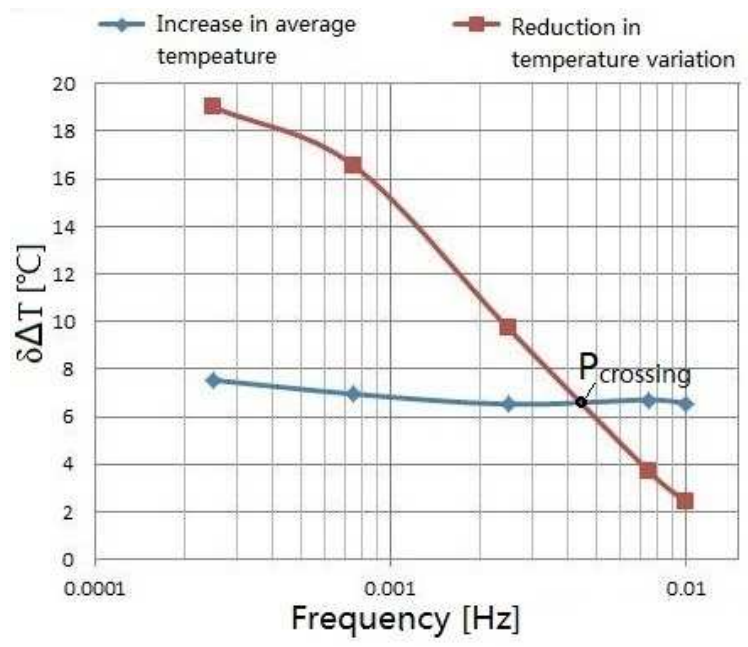

Figure 16: Experimental feedback control results in frequency domain (comparing to nominal cooling)

The experimental test with a more realistic load profile has been carried out to verify the proposed observer based controller's performance. Here different power dissipation steps was applied and the comparison with the situation without control is shown in Fig. 17. The results clearly show that the output temperature under designed control has faster response time and much smaller amplitude of temperature variations to step variations in the power dissipation profile. 


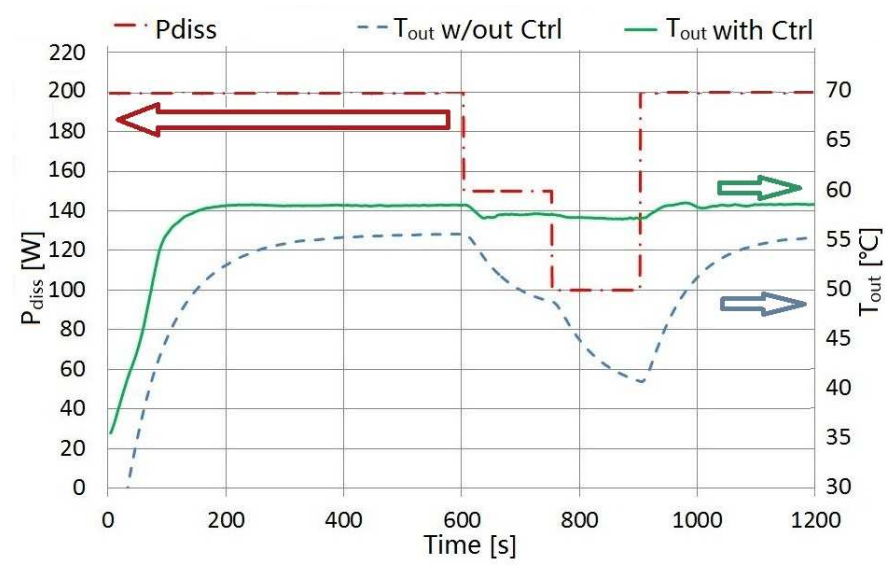

Figure 17: experimental results for control methodology/platform validation. Reported are: power dissipation profile (top dashed line); output temperature $\left(T_{\text {out }}\right)$ without control (fan at constant nominal maximum bias voltage/speed; bottom dashed line); output temperature $\left(T_{\text {out }}\right)$ with control.

\section{Discussion}

The designed controller can regulate the output temperature to the reference value under any power dissipation frequencies. However, when using fixed cooling without control, a higher power dissipation frequency will result in a smaller thermal cycling. Therefore, the reduction in $\delta T$ will be very small for very high frequency power dissipation so that the reliability improvement will be very limited. But, this only valid when the reference temperature is a fixed value. For example, as shown in Figure. 18, for any certain frequency, assuming the power profile is sinusoidal, the average temperature $T_{m}=\left(T_{\max }+T_{\min }\right) / 2$ and the controlled temperature is set to the maximum temperature for a certain power frequency, that is, $T_{\max }=T_{\min }+\delta T=T_{m}+1 / 2 \delta T$. So, the increase in $T_{m}$ is up to $1 / 2 \delta T$. Since the temperature reduction in $\delta T$ is always two times of the increase in $T_{m}$, a better reliability can be achieve. Therefore, if the reference temperature for the control system can be set properly according to the power frequency to $T_{\text {max }}$, the location of $P_{\text {crossing }}$ can move further to a higher frequency.

Although only the forced air convection cooling was used in this paper, cooling principle can be easily applied to different cooling solutions (e.g.,liquid cooling). The equivalent thermal resistance $R_{\text {cooling }}$ can be controlled similarly by adjusting the corresponding cooling parameters (e.g., 


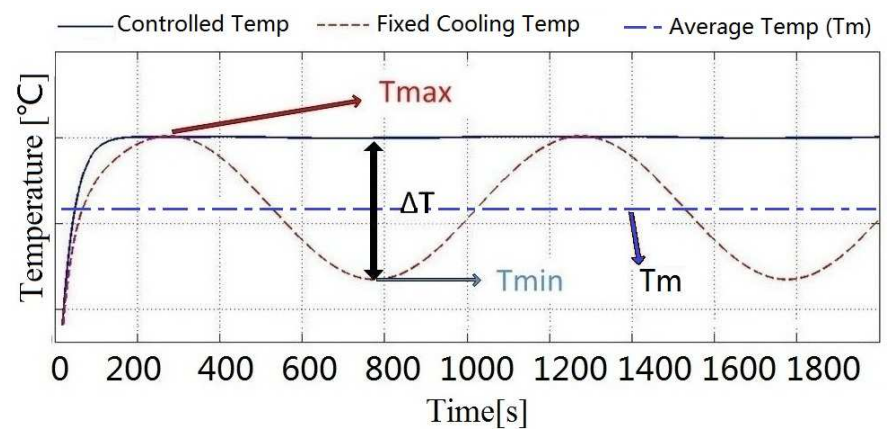

Figure 18: temperature comparison schematic plot

$\left.V_{\text {cooling }}\right)$. To demonstrate better the reliability improvement, the designed cooling strategy will be applied to real power systems again with realistic load profiles.

In experiment, when ambient temperature is set to change rapidly, the temperature inside the thermal chamber may be distributed non-uniformly as hot air flows are provided mainly by one fan inside the chamber. The thermocouple should be located carefully and precisely as any offset can be considerable when compared to the dimension of the thermal layers and that can affect the observer's accuracy.

\section{Conclusions}

In this paper we proposed a full-order observer based temperature control cooling strategy for power electronics cooling, aimed at reducing temperature variation under varying load conditions (e.g. varying power dissipation and ambient temperature profiles). The theoretical analysis and experimental results have shown a good agreement for the designed controller in reducing temperature variation. Within a meaningful load profile, the controlled temperature variation reduction can be kept larger than the increased average temperature so that more cycles to failures can be achieved. Therefore, a contribution to power devices' reliability and lifetime can be made. Future work will concentrate on implementing the designed control strategy onto a real power assembly with more realistic power load profiles. FEA (Finite Element Analysis) method will be involved in designing a more accurate thermal model and the tested module will be examined by scanning acoustic microscope so that a more visualised difference in reliability improvement can be achieved. 


\section{References}

[1] W. Wu, M. Held, P. Jacob, P. Scacco, A. Birolini, Thermal stress related packaging failure in power igbt modules, in: Power Semiconductor Devices and ICs, 1995. ISPSD '95. Proceedings of the 7th International Symposium on, pp. $330-334$.

[2] M. Ciappa, Selected failure mechanisms of modern power modules, Microelectronics Reliability 42 (2002) 653 - 667.

[3] T. Nakagawa, Replacement policies for a unit with random and wearout failures, Reliability, IEEE Transactions on R-29 (1980) 342 -344.

[4] D. Badenius, Random failure, Reliability, IEEE Transactions on R-19 (1970) $86-88$.

[5] X. Li, J. Qin, J. Bernstein, Compact modeling of mosfet wearout mechanisms for circuit-reliability simulation, Device and Materials Reliability, IEEE Transactions on 8 (2008) $98-121$.

[6] X. Wang, A. Castellazzi, P. Zanchetta, Regulated cooling for reduced thermal cycling of power devices, in: Power Electronics and Motion Control Conference (IPEMC), 2012 7th International, volume 1, pp. $238-244$.

[7] B. Ji, V. Pickert, B. Zahawi, M. Zhang, In-situ bond wire health monitoring circuit for igbt power modules, in: Power Electronics, Machines and Drives (PEMD 2012), 6th IET International Conference on, pp. 1 -6 .

[8] M. Bouarroudj, Z. Khatir, J. Ousten, F. Badel, L. Dupont, S. Lefebvre, Degradation behavior of 600v-200a igbt modules under power cycling and high temperature environment conditions, Microelectronics Reliability 47 (2007) 1719-1724.

[9] V. Smet, F. Forest, J. Huselstein, F. Richardeau, Z. Khatir, S. Lefebvre, M. Berkani, Ageing and failure modes of igbt modules in hightemperature power cycling, Industrial Electronics, IEEE Transactions on 58 (2011) $4931-4941$. 
[10] X. Perpina, J.-F. Serviere, J. Urresti-Ibanez, I. Cortes, X. Jorda, S. Hidalgo, J. Rebollo, M. Mermet-Guyennet, Analysis of clamped inductive turnoff failure in railway traction igbt power modules under overload conditions, Industrial Electronics, IEEE Transactions on 58 (2011) 2706 -2714 .

[11] G. Buiatti, J. Martin-Ramos, C. Garcia, A. Amaral, A. Cardoso, An online and noninvasive technique for the condition monitoring of capacitors in boost converters, Instrumentation and Measurement, IEEE Transactions on 59 (2010) $2134-2143$.

[12] L. Ngwendson, M. Sweet, E. Narayanan, An overview of the recent developments in high-voltage power semiconductor mos-controlled bipolar devices, in: Bipolar/BiCMOS Circuits and Technology Meeting, 2009. BCTM 2009. IEEE, pp. 198 -205.

[13] A. Morozumi, K. Yamada, T. Miyasaka, S. Sumi, Y. Seki, Reliability of power cycling for igbt power semiconductor modules, Industry Applications, IEEE Transactions on 39 (2003) 665 - 671.

[14] S. Igarashi, H. Kakiki, Y. Nishimura, T. Goto, Design of high reliability packaging for fuji high power module, in: Electrical Machines and Systems (ICEMS), 2011 International Conference on, pp. 1 -6.

[15] U. Scheuermann, Reliability challenges of automotive power electronics, Microelectronics Reliability 49 (2009) 1319 - 1325.

[16] D. Murdock, J. Torres, J. Connors, R. Lorenz, Active thermal control of power electronic modules, Industry Applications, IEEE Transactions on 42 (2006) 552-558.

[17] R. Skuriat, C. M. Johnson, Direct substrate cooling of power electronics, Integrated Power Systems (CIPS), 2008 5th International Conference on (2008) $1-5$.

[18] U. Scheuermann, U. Hecht, Power cycling lifetime of advanced power modules for different temperature swings, PCIM Nuremberg (2002) 5964 . 
[19] A. Castellazzi, Comprehensive compact models for the circuit simulation of multichip power modules, Power Electronics, IEEE Transactions on 25 (2010) $1251-1264$.

[20] M. Cotorogea, Physics-based spice-model for igbts with transparent emitter, Power Electronics, IEEE Transactions on 24 (2009) 2821 -2832.

[21] G. Franklin, J. Powell, A. Emami-Naeini, J. Powell, Feedback control of dynamic systems, volume 3, Addison-Wesley Reading, MA, 1994.

[22] R. Chassaing, DSP applications using C and the TMS320C6x DSK, volume 11, Wiley-Interscience, 2002. 